\title{
Graph Cuts in Vision and Graphics: Theories and Applications
}

\author{
Yuri Boykov and Olga Veksler \\ Computer Science, The University of Western Ontario, London, Canada \\ [yuri,olga]@csd.uwo.ca
}

\begin{abstract}
Combinatorial min-cut algorithms on graphs emerged as an increasingly useful tool for problems in vision. Typically, the use of graphcuts is motivated by one of the following two reasons. Firstly, graph-cuts allow geometric interpretation; under certain conditions a cut on a graph can be seen as a hypersurface in N-D space embedding the corresponding graph. Thus, many applications in vision and graphics use min-cut algorithms as a tool for computing optimal hypersurfaces. Secondly, graphcuts also work as a powerful energy minimization tool for a fairly wide class of binary and non-binary energies that frequently occur in early vision. In some cases graph cuts produce globally optimal solutions. More generally, there are iterative graph-cut based techniques that produce provably good approximations which (were empirically shown to) correspond to high-quality solutions in practice. Thus, another large group of applications use graph-cuts as an optimization technique for low-level vision problems based on global energy formulations.

This chapter is intended as a tutorial illustrating these two aspects of graph-cuts in the context of problems in computer vision and graphics. We explain general theoretical properties that motivate the use of graph cuts, as well as, show their limitations.
\end{abstract}

\section{Introduction}

Graph cuts remain an area of active research in the vision and graphics communities. Besides finding new applications, in the last years researchers discovered and rediscovered interesting links connecting graph cuts with other combinatorial algorithms (dynamic programming, shortest paths [6,22]), Markov random fields, statistical physics, simulated annealing and other regularization techniques [17, 10, 19], sub-modular functions [25], random walks and electric circuit theory [15, 16], Bayesian networks and belief propagation [37], integral/differential geometry, anisotropic diffusion, level sets and other variational methods [36, 7, 2, 22].

Graph cuts proved to be a useful multidimensional optimization tool which can enforce piecewise smoothness while preserving relevant sharp discontinuities. This paper is mainly intended as a survey of existing literature and a tutorial on graph cuts in the context of vision and graphics. We present some basic background information on graph cuts and discuss major theoretical results, some fairly new and some quite old, that helped to reveal both strengths and limitations of these surprisingly versatile combinatorial algorithms. This chapter does 
not provide any new research results, however, some applications are presented from a point of view that may differ from the previous literature.

The organization of this chapter is as follows. Chapter 2 provides necessary background information and terminology. In their core, combinatorial mincut/max-flow algorithms are binary optimization methods. Chapter 3 presents a simple binary problem that can help to build basic intuition on using graph cuts in computer vision. Then, graph cuts are discussed as a general tool for exact minimization of certain binary energies.

Most publications on graph cuts in vision and graphics show that, despite their binary nature, "graph cuts" offer significantly more than "binary energy minimization". Chapter 4 shows that graph cuts provide a viable geometric framework for approximating continuous hypersurfaces on N-dimensional manifolds. This geometric interpretation of graph cuts is widely used in applications for computing globally optimal separating hypersurfaces. Finally, Chapter 5 presents generalized (non-binary) graph cuts techniques applicable to exact or approximate minimization of multi-label energies. In the last decade, such nonbinary graph cut methods helped to significantly raise a "plank" for what is considered a good quality solution in many early vision problems.

\section{Graph Cuts Basics}

First of all, we introduce the basic terminology. Let $\mathcal{G}=\langle\mathcal{V}, \mathcal{E}\rangle$ be a graph which consists of a set of nodes $\mathcal{V}$ and a set of directed edges $\mathcal{E}$ that connect them. The nodes set $\mathcal{V}=\{s, t\} \cup \mathcal{P}$ contains two special terminal nodes, which are called the source, $s$, and the sink, $t$, and a set of non-terminal nodes $\mathcal{P}$. In Figure 1(a) we show a simple example of a graph with the terminals $s$ and $t$. Such N-D grids are typical for applications in vision and graphics.

Each graph edge is assigned some nonnegative weight or cost $w(p, q)$. A cost of a directed edge $(p, q)$ may differ from the cost of the reverse edge $(q, p)$. An edge is called a $t$-link if it connects a non-terminal node in $\mathcal{P}$ with a terminal. An edge is called a $n$-link if it connects two non-terminal nodes. A set of all (directed) n-links will be denoted by $\mathcal{N}$. The set of all graph edges $\mathcal{E}$ consists of n-links in $\mathcal{N}$ and t-links $\{(s, p),(p, t)\}$ for non-terminal nodes $p \in \mathcal{P}$. In Figure 1 t-links are shown in red and blue, while n-links are shown in yellow.

\subsection{The Min-Cut and Max-Flow Problem}

An $s / t$ cut $C$ (sometimes we just call it a $c u t$ ) is a partitioning of the nodes in the graph into two disjoint subsets $\mathcal{S}$ and $\mathcal{T}$ such that the source $s$ is in $\mathcal{S}$ and the sink $t$ is in $\mathcal{T}$. Figure 1(b) shows one example of a cut. The cost of a cut $C=\{\mathcal{S}, \mathcal{T}\}$ is the sum of costs/weights of "boundary" edges $(p, q)$ such that $p \in \mathcal{S}$ and $q \in \mathcal{T}$. If $(p, q)$ is a boundary edge, then we sometimes say that cut $C$ severs edge $(p, q)$. The minimum cut problem is to find a cut that has the minimum cost among all cuts. 


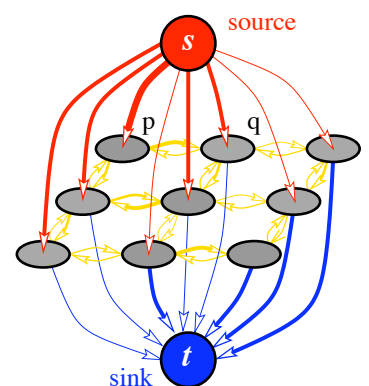

(a) A graph $\mathcal{G}$

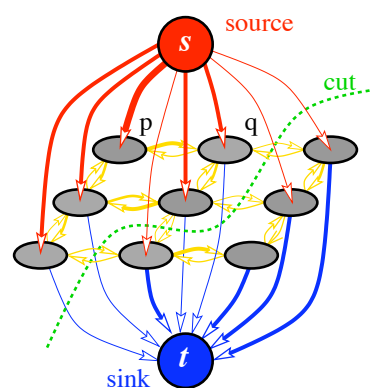

(b) A cut on $\mathcal{G}$

Fig. 1. Graph construction in Greig et. al. [17]. Edge costs are reflected by thickness.

One of the fundamental results in combinatorial optimization is that the minimum $s / t$ cut problem can be solved by finding a maximum flow from the source $s$ to the sink $t$. Speaking informally, maximum flow is the maximum "amount of water" that can be sent from the source to the sink by interpreting graph edges as directed "pipes" with capacities equal to edge weights. The theorem of Ford and Fulkerson [12] states that a maximum flow from $s$ to $t$ saturates a set of edges in the graph dividing the nodes into two disjoint parts $\{\mathcal{S}, \mathcal{T}\}$ corresponding to a minimum cut. Thus, min-cut and max-flow problems are equivalent. In fact, the maximum flow value is equal to the cost of the minimum cut.

\subsection{Algorithms for the Min-Cut and Max-Flow Problem}

There are many standard polynomial time algorithms for min-cut/max-flow[11]. These algorithms can be divided into two main groups: "push-relabel" style methods [14] and algorithms based on augmenting paths. In practice the pushrelabel algorithms perform better for general graphs. In vision applications, however, the most common type of a graph is a two or a higher dimensional grid. For the grid graphs, Boykov and Kolmogorov [8] developed a fast augmenting path algorithm which often significantly outperforms the push relabel algorithm. Furthermore, its observed running time is linear.

While the (sequential) algorithm in [8] is very efficient, with the execution time of only a few seconds for a typical problem, it is still far from real time. A possible real time solution may come from a GPU acceleration that has become popular for improving the efficiency of algorithms allowing parallel implementations on pixel level. Note that push-relabel algorithm can be run in parallel over graph nodes [14]. In the context of image analysis problems, graph nodes typically correspond to pixels. Thus, pixel based GPU architecture is a seemingly perfect match for accelerating push-relabel algorithm for computing graph cuts in vision and graphics. This is a very promising direction for getting applications of graph cuts up to real time. 


\section{Graph Cuts for Binary Optimization}

In this section we concentrate on graph cuts as a binary optimization tool. In fact, underlying min-cut/max-flow algorithms are inherently binary techniques. Thus, binary problems constitute the most basic case for graph cuts. In Section 3.1 we discuss the earliest example where graph cuts were used in vision, which also happens to be a particularly clear binary problem. This example illustrates that graph cuts can effectively enforce spacial coherence on images. Section 3.2 presents the general case of binary energy minimization with graph cuts.

\subsection{Example: Binary Image Restoration}

The earliest use of graph cuts for energy minimization in vision is due to Greig et.al. [17]. They consider the problem of binary image restoration. Given a binary image corrupted by noise, the task is to restore the original image. This problem can be formulated as a simple optimization over binary variables corresponding to image pixels. In particular, [17] builds a graph shown in Figure 1(a) where non-terminal nodes $p \in \mathcal{P}$ represent pixels while terminals $s$ and $t$ represent two possible intensity values. To be specific, source $s$ will represent intensity 0 and sink $t$ will represent intensity 1 . Assume that $I(p)$ is the observed intensity at pixel $p$. Let $D_{p}(l)$ be a fixed penalty for assigning to pixel $p$ some "restored intensity" label $l \in\{0,1\}$. Naturally, if $I(p)=0$ then $D_{p}(0)$ should be smaller than $D_{p}(1)$, and vice versa. To encode these "observed data" constraints, we create two t-links for each pixel node in Figure 1. Weight of t-link $(s, p)$ is set to $D_{p}(1)$ and weight of $(p, t)$ is $D_{p}(0)$. Even though t-link weights should be non-negative, restriction $D_{p} \geq 0$ for data penalties is not essential.

Now we should add regularizing constraints that help to remove image noise. Such constraints enforce spacial coherence between neighboring pixels by minimizing discontinuities between them. In particular, we create n-links between neighboring pixels using any (e.g. 4- or 8-) neighborhood system. The weight of these n-links is set to a smoothing parameter $\lambda>0$ that encourages minimum cut to sever as few n-links as possible.

Remember that a cut $C$ (Figure $1(\mathrm{~b})$ ) is a binary partitioning of the nodes into subsets $\mathcal{S}$ and $\mathcal{T}$. A cut can be interpreted as a binary labeling $f$ that assigns labels $f_{p} \in\{0,1\}$ to image pixels: if $p \in \mathcal{S}$ then $f_{p}=0$ and if $p \in \mathcal{T}$ then $f_{p}=1$. Obviously, there is a one-to-one correspondence between cuts and binary labelings of pixels. Each labeling $f$ gives a possible image restoration result.

Consider the cost of an arbitrary cut $C=\{\mathcal{S}, \mathcal{T}\}$. This cost includes weights of two types of edges: severed t-links and severed n-links. Note that a cut severs exactly one t-link per pixel; it must sever t-link $(p, t)$ if pixel $p$ is in the source component $p \in \mathcal{S}$ or t-link $(s, p)$ if pixel $p$ is in the sink component $p \in \mathcal{T}$. Therefore, each pixel $p$ contributes either $D_{p}(0)$ or $D_{p}(1)$ towards the t-link part of the cut cost, depending on the label $f_{p}$ assigned to this pixel by the cut. The cut cost also includes weights of severed n-links $(p, q) \in \mathcal{N}$. Therefore,

$$
|C|=\sum_{p \in \mathcal{P}} D_{p}\left(f_{p}\right)+\sum_{\substack{(p, q) \in \mathcal{N} \\ p \in \mathcal{S}, q \in \mathcal{T}}} w(p, q)
$$


The cost of each $C$ defines the "energy" of the corresponding labeling $f$ :

$$
E(f):=|C|=\sum_{p \in \mathcal{P}} D_{p}\left(f_{p}\right)+\lambda \cdot \sum_{(p, q) \in \mathcal{N}} \mathcal{I}\left(f_{p}=0, f_{q}=1\right)
$$

where $\mathcal{I}(\cdot)$ is the identity function giving 1 if its argument is true and 0 otherwise. Stated simply, the first term says that pixel labels $f_{p}$ should agree with the observed data while the second term penalised discontinuities between neighboring pixels. Obviously, minimum cut gives labeling $f$ minimizing energy (1).

Note that parameter $\lambda$ weights the relative importance of the data constraints and the regularizing constraints. Note that if $\lambda$ is very small, optimal labeling assigns each pixel $p$ a label $f_{p}$ that minimizes its own data cost $D_{p}\left(f_{p}\right)$. In this case, each pixel chooses its own label independently from the other pixels. If $\lambda$ is big, then all pixels must choose one label that has a smaller average data cost. For intermediate values of $\lambda$, optimal labeling $f$ should correspond to a balanced solution with compact spatially coherent clusters of pixels who generally like the same label. Noise pixels, or outliers, should conform to their neighbors.

Before [17], exact minimization of energies like (1) was not possible. Researches still liked them, but had to approach them with iterative algorithms like simulated annealing [13]. In fact, Greig et.al. published their result mainly to show that in practice simulated annealing reaches solutions very far from the global minimum even in simple binary cases. Unfortunately, the result of Greig et.al. remained unnoticed in the vision community for almost 10 years probably because the binary image restoration looked too restrictive as an application.

\subsection{General Case of Binary Energy Minimization}

In general, graph construction as in Figure 1 can be used for other binary "labeling" problems. Suppose we are given a penalty $D_{p}(l)$ that pixel $p$ incurs when assigned label $l \in \mathcal{L}=\{0,1\}$ and we need to find a spatially coherent binary labeling of the whole image. We may wish to enforce spacial regularization via some global energy function that generalizes (1)

$$
E(f)=\sum_{p \in \mathcal{P}} D_{p}\left(f_{p}\right)+\sum_{(p, q) \in \mathcal{N}} V_{p q}\left(f_{p}, f_{q}\right)
$$

The question is: can we find a globally optimal labeling $f$ using some graph cuts construction? There is a definitive answer to this question for the case of binary labelings. According to [25], a globally optimal binary labeling for (2) can be found via graph cuts if and only if the pairwise interaction potential $V_{p q}$ satisfies

$$
V_{p q}(0,0)+V_{p q}(1,1) \leq V_{p q}(0,1)+V_{p q}(1,0)
$$

which is called regularity condition. The theoretical result in [25] is constructive and they show the corresponding graph. It has the same form as the graph of Greig et.al. in Figure 1, however, edge weights are derived differently. 


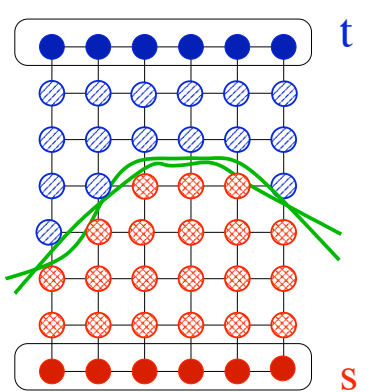

(a) a cut on a $2 \mathrm{D}$ grid
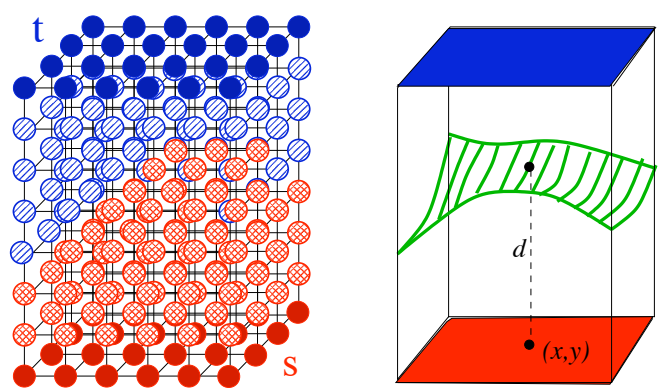

(b) a cut and a separating hypersurface in 3D

Fig. 2. s-t cut on a grid corresponds to binary partitioning of N-D space where the grid is embedded. Such space partitioning may be visualized via a separating hypersurface. As shown in (a), multiple hypersurfaces may correspond to the same cut. However, such hypersurfaces become indistinguishable as the grid gets finer.

\section{Graph Cuts as Hypersurfaces}

Solution of many problems in vision, image processing and graphics can be represented in terms of optimal hypersurfaces. This section describes a geometric interpretation of graph-cuts as hypersurfaces in N-D manifolds that makes them an attractive framework for problems like image segmentation, restoration, stereo, photo/video editing, texture synthesis, and others.

We show a basic idea allowing to view s-t cuts as hypersurfaces, discuss interesting theories that make various connections between discrete graph cuts and hypersurfaces in continuous spaces, and provide a number of recently published examples where hypersurface view of graph cuts found interesting applications in computer vision, medical imaging, or graphics.

\subsection{Basic idea}

Consider two simple examples in Figure 2. Through out Section 4 we assume that a graph has no "soft" t-links, so that the source and the sink terminals are directly connected only to some of the graph nodes via infinity cost t-links. In fact, all nodes hardwired to two terminals can be effectively treated as multiple sources and multiple sinks that have to be separated by a cut. Figure 2 shows these sources and sinks in dark red and dark blue colors. Such sources and sinks provide hard constraints or boundary conditions for graph cuts; any feasible cut must separate sources from sinks. Other nodes are connected to the sources and sinks via n-links.

Without loss of generality (see Section 4.2), we can concentrate on feasible cuts that partition the simple 4- and 6- nearest neighbor grid-graphs in Figure 2 into two connected subsets of nodes: source component and sink component. Continuous 2D and 3D manifolds where the grid nodes are embedded can be 
split into two disjoint contiguous regions containing the sinks and the sources, correspondingly. A boundary between two such regions are separating hypersurfaces shown in green color. As illustrated in Figure 2(a), there are many separating hypersurfaces that correspond to the same cut. They should all correctly separate the grid nodes of the source and the sink components, but they can "freely move" in the space between the grid nodes. Without getting into mathematical details, we will identify a class of all hypersurfaces corresponding to a given cut with a single hypersurface. In particular, we can choose a hypersurface that follows boundaries of "grid cells", or we can choose "the smoothest" hypersurface. Note that the finer the grid, the harder it is to distinguish two separating hypersurfaces corresponding to the same cut.

Thus, any feasible cut on a grid in Figure 2 corresponds to a separating hypersurface in the embedding continuous manifold. Obviously, the opposite is also true; any separating hypersurface corresponds to a unique feasible cut. Generalization of examples in Figure 2 would establish correspondence between $s-t$ graph-cuts and separating hypersurfaces in case of "fine" locally connected grids embedded in N-D spaces. Following ideas in [7], one can set a cost (or area) of each continuous hypersurface based on the cost of the corresponding cut. This defines a cut metric introduced in [7] for continuous N-D manifold embedding a graph. By changing weights of n-links at graph nodes located in any particular point in space, one can tune local costs of all separating hypersurfaces that pass through such locations. In practical applications cut metric can be easily tuned to attract (repel) hypersurfaces to (from) certain locations on N-D manifolds. Cut metric is a simple and yet, sufficiently general tool. In particular, according to [7] cut metric on 2D and 3D manifolds can approximate any given continuous Riemannian metric. Finally, standard combinatorial algorithms for computing minimum cost $s-t$ cuts (see Section 2.2) become numerical tools for extracting globally optimal separating hypersurfaces.

\subsection{Topological properties of graph cuts}

The adjective "separating" implies that a hypersurface should satisfy certain hard constraints or boundary conditions; it should separate source and sink grid cells (seeds). Note that there are many freedoms in setting boundary conditions for graph cuts. Depending on hard constraints, topological properties of separating hypersurfaces corresponding to $s-t$ cuts may vary.

For example, we can show that the boundary conditions in Figure 2 guarantee that any feasible cut corresponds to topologically connected separating hypersurface. For simplicity, we assume that our graphs are connected, that is, there are no "islands" of disconnected nodes. In Figure 2 all source and all sink nodes form two connected components. In such cases a minimum cost cut must partition the graph into exactly two connected subsets of nodes; one containing all sources and the other containing all sinks. Assuming that the minimum cost cut creates three or more connected components implies that some of these components contain neither sources, nor sinks. This contradicts minimality of 


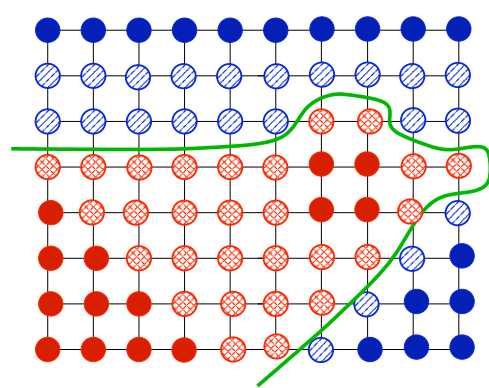

(a) connected source segment

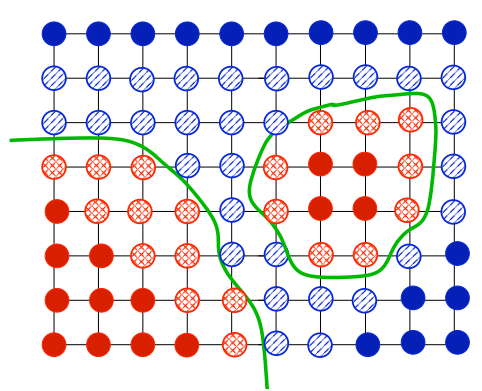

(b) disjoint source segments

Fig. 3. Separating hypersurfaces can have different topological properties for the same set of hard constraints. Separating hypersurfaces in (a) and (b) correspond to two distinct feasible $s-t$ cuts. Min-cut/max-flow algorithms compute globally optimal hypersurface/cut without any restrictions on its topological properties as long as the sources and the sinks are separated.

the cut; linking any "no-source/no-sink" subset back to the graph corresponds to a smaller cost feasible cut.

Examples in Figure 3 illustrates different topological properties for separating hypersurfaces in more general cases where multiple disjoint components of sources and sinks (seeds) are present. Note that feasible $s-t$ cuts may produce topologically different separating hypersurfaces for the same set of boundary conditions.

In fact, controlling topological properties of separating hypersurfaces by setting up appropriate hard constraints is frequently a key technical aspect of applications using graph cuts. As discussed in Section 4.3, appropriate positioning of sources and sinks is not the only tool to achieve desired topology. As shown in Figure 4, certain topological properties of separating hypersurfaces can be enforced via infinity cost n-links.

\subsection{Applications of graph cuts as hypersurfaces}

Below we consider several examples from recent publications where graph cuts are used as a method for extracting optimal hypersurfaces with desired topological properties.

Methods for object extraction $[6,5,30,41]$ take full advantage of topological freedom of graph-cut based hypersurfaces. In particular, they allow to segment objects of arbitrary topology. The basic idea is to set as sources (red seeds) some image pixels that are known (a priori) to belong to an object of interest and to set as sinks (blue seeds) some pixels that are known to be in the background. A separating hypersurface should coincide with a desirable object boundary separating object (red) seeds from background (blue) seeds, as demonstrated in Figure 3. Cut metric can be set to reflect on image gradient. Pixels with high 
image gradients would imply low cost of local n-links and vice-versa. Then, minimal separating hypersurfaces tend to adhere to object boundaries with hight image gradients. Another practical strength of object extraction methods based on graph cuts is that they provide practical solutions for organ extraction problems in N-D medical image analysis [6]. One limitation of this approach to object extraction is that it may suffer from a bias to "small cuts" which could be often resolved with proper constraining of solution space.

Stereo was one of the first applications in computer vision where graph cuts were successfully applied as a method for optimal hypersurface extraction. Two teams, Roy\&Cox $[32,31]$ and Ishikawa\&Geiger [20], almost simultaneously proposed two different formulations of stereo problem where disparity maps are interpreted as separating hypersurfaces on certain 3D manifolds. Their key technical contribution was to show that disparity maps (as optimal hypersurfaces) can be efficiently computed via graph cuts.

For example, Roy\&Cox [32,31] proposed a framework for stereo where disparity maps are separating hypersurfaces on 3D manifolds similar to one in Figure 2(b). Points of this bounded rectangular manifold are interpreted as points in 3D "disparity space" corresponding to a pair of rectified stereo images. This disparity space is normally chosen with respect to one of the images, so that each 3D point with coordinates $(x, y, d)$ represents correspondence between pixel $(x, y)$ in the first stereo image and pixel $(x+d, y)$ in the second image. Then, solution of stereo problem is a hypersurface $d=f(x, y)$ on 3D manifold in Figure 2(b) that represents a disparity map assigning certain disparity $d$ to each pixel $(x, y)$ in the first image. Note that hypersurface $d=f(x, y)$ separates the bottom and the top (facets) of 3D manifold in Figure 2(b). Then, an optimal disparity map can be computed using graph cuts as an efficient discrete model for extracting minimal separating hypersurfaces.

According to [32], cut metric on 3D "disparity space" manifold in Figure 2(b) is set based on color consistency constraint between two stereo cameras. Weights of n-links at node $(x, y, d)$ are set as follows: if intensities of pixels $(x, y)$ and $(x+$ $d, y)$ in two cameras are similar then the likelihood that two pixels see the same $3 \mathrm{D}$ object point is high and the cost of n-links should be small. Later, $[21,31$, 9] suggested anisotropic cut metric where vertical n-links are based on the same likelihoods as above but horizontal n-links are fixed to a constant encouraging smoother disparity maps that avoid unnecessary disparity level jumps.

In general, separating hypersurfaces in Figure 2(b) can have folds that would make them inappropriate as disparity maps $d=f(x, y)$. If a minimum hypersurface computed via graph cuts has a fold then we did not find a feasible disparity map. Therefore, $[21,9]$ propose a set of hard constraints that make topological folds (see Figure 4(a)) prohibitively expensive. Note that additional infinity cost vertical n-links (directed down) make folds infeasible. This topological hard constraint take advantage of "directed" nature of graph cuts; a cost of a cut includes only severed directed edges that go from the (red) nodes in the source component to the (blue) nodes in the sink component. A cut with an illegal fold in Figure 4(a) includes one infinity cost n-link. 


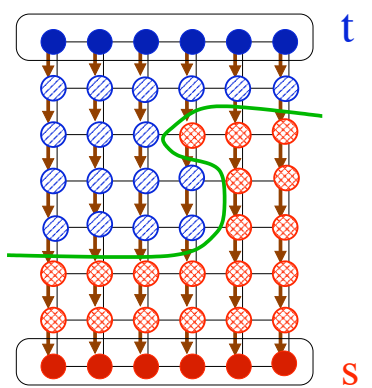

(a) Infeasible folding in $[32,31]$

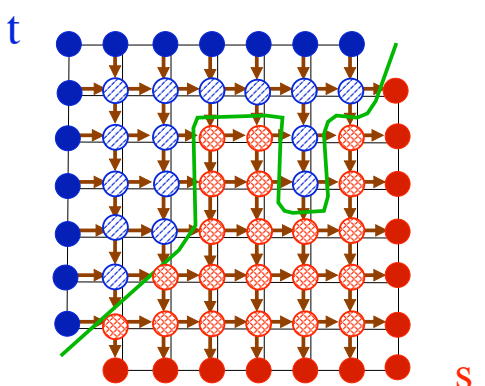

(b) Infeasible folding in [20]

Fig. 4. Graph-cuts approach allows to impose certain additional topological constraints on separating hypersurfaces, if necessary. For example, [21,9] proposed infinity cost directed n-links, shown in brown color in (a), that forbid folds on separating hypersurfaces in Figure 2. In particular, a hypersurface in Figure 2(b) without such folds corresponds to a disparity map $d=f(x, y)$ according to [32,31]. Also, [20] impose monotonicity/ordering constraint on their disparity maps by adding infinity cost directed n-links (in brown color) that make illegal topological folds shown in (b). For clarity, examples in (a) and (b) correspond to single slices of 3D manifolds in Figure 2(b) and 5(a).

Ishikawa\&Geiger [20] also solve stereo by computing optimal separating hypersurfaces on a rectangular 3D manifold. However, their interpretation of the manifold and boundary conditions are different. As shown in Figure 5(a), they interpret a separating hypersurface $z=f(x, y)$ as a "correspondence mapping" between pixels $p=(x, y)$ in the left image and pixels $q=(f(x, y), y)$ in the right image (of a rectified stereo pair). Assignment of correspondences may be ambiguous if a hypersurface has folds like one in Figure 4(b). In order to avoid ambiguity, [20] introduce monotonicity (or ordering) constraint that is enforced by directed infinity cost n-links shown in brown color. Note that a cut in Figure 4(b) severs two brown n-links that go from a (red) node in a source component to a (blue) node in a sink component. Thus, the cost of the cut is infinity and the corresponding separating hypersurface with a fold becomes infeasible.

Similar to [32,31], the cut metric on manifold in Figure 5(a) is based on color consistency constraint: a $3 \mathrm{D}$ points $(x, y, z)$ on the manifold has low n-link costs if intensity of pixel $(x, y)$ in the left image is close to intensity of pixel $(z, y)$ in the right image. Note that hyperplanes parallel to diagonal crossection (from bottom-left to top-right corners) of manifold in Figure 5(a) give correspondence mappings with constant stereo disparity/depth levels. Thus, spacial consistency of disparity/depth map can be enforced with anisotropic cut metric where diagonal n-links (from left-bottom to right-top corner) are set to a fixed constant representing penalty for jumps between disparity levels.

Another interesting example of graph-cuts/hypersurface framework is a method for video texture synthesis in [26]. The technique is based on computing a seamless transition between two video clips as illustrated in Figure 5(b). Two clips 


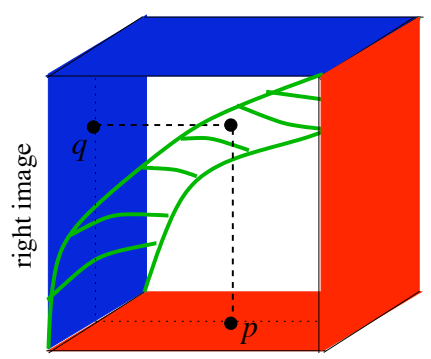

left image

(a) Hypersurface as correspondence

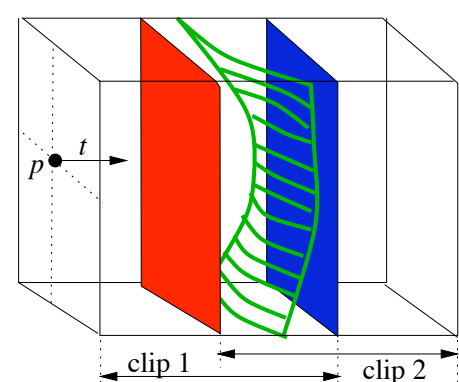

(b) Hypersurface separates two video clips

Fig. 5. Two more examples of graph cuts as separating hypersurfaces. Formulation of stereo problem in [20] computes pixel correspondences represented by a separating hypersurface on a 3D manifold in (a). A smooth transition between two video clips is performed in [26] via graph cuts computing globally optimal separating hypersurface in a $3 \mathrm{D}$ region of overlap between two clips in (b).

are overlapped in 3D (pixel-time) space creating a bounded rectangular manifold where transition takes place. A point in this manifold can be described by $3 \mathrm{D}$ coordinates $(x, y, t)$ where $p=(x, y)$ is a pixel and $t$ is time or video frame number. The transition is represented by a separating hypersurface $t=f(x, y)$ that specifies for each pixel when to switch from clip 1 to clip 2. During transition a frame may have a mix of pixels from each clip. The method in [26] suggest a specific cut metric that for each point $(x, y, t)$ in the overlap region depends on intensity difference between two clips. Small difference indicates a good moment (in space and time) for seamless transition between the clips and n-links at such $(x, y, t)$ points are assigned a low cost. Note that "seamless transition" is a purely visual effect and it may be achieved with any separating hypersurface in Figure 5(b). In this case there is no real need to avoid hypersurfaces with "folds" which would simply allow pixels to switch between clip 1 and clip 2 a few times.

\subsection{Theories connecting graph-cuts and hypersurfaces in $R^{n}$}

In this section we discuss a number of known results that established theoretically solid connections between cuts on discrete graphs and hypersurfaces in continuous spaces. It has been long argued in computer vision literature that discrete algorithms on graphs, including graph cuts, may suffer from metrication artifacts. Indeed, 4- and 6- nearest neighbor connections on 2D and 3D grids may produce "blocky" segments. Such geometric artifacts are due to "Manhattan distance" metrication errors. It turns out that such errors can be easily corrected resolving the long-standing criticism of graph cuts methods. Boykov\&Kolmogorov [7] showed that regular grids with local neighborhood systems of higher order can produce a cut metric that approximates any continuous Riemannian metric with arbitrarily small error. Using powerful results from integral geometry, [7] shows 
that weights of n-links from a graph node embedded at point $p$ of continuous N-D manifold are solely determined by a given $N \times N$ positive-definite matrix $D(p)$ that defines local metric/distance properties at point $p$ according to principles of Riemannian geometry. This result is quite intuitive as weights of n-links at this graph node define local measure for area/distance for hypersurfaces according to the corresponding cut metric. It is also interesting that results in [7] apply to arbitrary Riemannian metrics including anisotropic cases where local metric could be direction-sensitive.

So far in Section 4 we followed the general approach of [7] where hypersurfaces on N-D manifolds have implicit representation via cuts on embedded graphs. As illustrated in Figure 2, a cut only "implies" a separating hypersurface. A specific hypersurface can be obtained through additional conventions, as discussed in Section 4.1. More recently, [22] proposed an explicit approach to hypersurface representation by graph cuts that, in a way, is dual to [7]. The basic idea in [22] is to bisect a bounded N-D manifold with a large number of (random) hyperplanes. These hyperplanes divide the manifold into small cells (polyhedra) which can be thought of as irregular voxels. Then, [22] build an irregular "random-grid" graph where each cell is represented by a node. Two cells are connected by an n-link if and only if they touch through a common facet. Clearly, there is a one-to-one correspondence between a set of all n-links on the graph and a set of all facets between cells. A cut on this graph explicitly represents a unique hypersurface formed by facets corresponding to severed n-links. Obviously, a cost of any cut will be equal to the area of the corresponding hypersurface (in any metric) if weights of each n-link is equal to the area of the corresponding facet (in that metric). Thus, the model for representing hypersurfaces via graph-cuts in [22] can be applied to any metric. In their case, min-cut/max-flow algorithms will compute a minimum separating hypersurface among all explicitly represented hypersurfaces satisfying given boundary conditions.

Cuts on a graph in [22] represent only a subset of all possible hypersurfaces on an embedding manifold. If one keeps bisecting this bounded manifold into finer cells then the number of representable hypersurfaces increases. [22] proves that bisecting the manifold with a countably infinite number of random hyperplanes would generate small enough cells so that their facets can represent any continuous ${ }^{1}$ hypersurface with an arbitrarily small error. This demonstrates that their approach to graph-cut/hypersurface representation is also theoretically solid.

Intuitively speaking, theoretical results in [7] and [22] imply that both approaches to representing continuous hypersurfaces via discrete graph cuts models have reasonable convergence properties and that minimum cost cuts on finer graphs "in the limit" produce a minimum separating hypersurfaces for any given metric. Results such as [7] and [22] also establish a link between graph cuts and variational methods such as level-sets $[35,28,34,29]$ that are also widely used for image segmentation.

There is (at least) one more interesting theoretical result linking graph cuts and hypersurfaces in continuous spaces that is due to G. Strang [36]. This result

\footnotetext{
1 piece-wise twice differentiable, see [22] for more details.
} 
was established more than 20 years ago and it gives a view somewhat different from $[7,22]$. Strang describes a continuous analogue of the min-cut/max-flow paradigm. He shows that maximum flow problem can be redefined on a bounded continuous domain $\Omega$ in the context of a vector field $\bar{f}(p)$ representing the speed of a continuous stream/flow. A constraint on discrete graph flow that comes from edge capacities is replaced by a "speed limit" constraint $|\bar{f}(p)| \leq c(p)$ where $c$ is a given non-negative scalar function ${ }^{2}$. Discrete flow conservation constraint for nodes on a graph has a clear continuous interpretation as well: a continuous stream/flow is "preserved" at points inside the domain if vector field $\bar{f}$ is divergence-free $\operatorname{div} \bar{f}=0$. Strang also gives appropriate definition for sources and sinks on the boundary of the domain ${ }^{3}$. Then, the continuous analogue of the maximum flow problem is straightforward: find a maximum amount of water that continuous stream $\bar{f}$ can take from sources to sinks across the domain while satisfying all the constraints.

The main topic of this sections connects to [36] as follows. Strang defines a "real" cut on $\Omega$ as a hypersurface $\gamma$ that divides the domain into two subsets. The minimum cut should separate sources and sinks and have the smallest possible $\operatorname{cost} \int_{\gamma} c$ which can be interpreted as a length of hypersurface $\gamma$ in isotropic metric defined by a scalar function $c$. Strang also establishes duality between continuous versions of minimum cut and maximum flow problems that is analogous to the discrete version established by Ford and Fulkerson [12]. On a practical note, a recent work by Appleton\&Talbot [2] proposed a finite differences approach that, in the limit, converges to a globally optimal solution of continuous mincut/max-flow problem defined by Strang. Note, however, that they use graph cuts algorithms to "greatly increase the speed of convergence".

\section{Generalizing Graph Cuts for Multi-Label Problems}

In this section, we show that even though graph cuts provide an inherently binary optimization, they can be used for multi-label energy minimization. In some cases, minimization is exact, but in more interesting cases only approximate minimization is possible. There is a direct connection between the exact multi-label optimization and a graph cut as a hypersurface interpretation of Section 4 . We begin by stating the general labeling problem, then in Section 5.1 we describe the case when optimization can be performed exactly. Finally, Section 5.2 describes the approximate minimization approaches and their quality guarantees.

Many problems in vision and graphics can be naturally formulated in terms of multi-label energy optimization. Given a set of sites $\mathcal{P}$ which represent pixels/voxels, and a set of labels $\mathcal{L}$ which may represent intensity, stereo disparity, a motion vector, etc., the task is to find a labeling $f$ which is a mapping from

\footnotetext{
${ }^{2}$ More generally, it is possible to set an anisotropic "speed limit" constraint $\bar{f}(p) \in c(p)$ where $c$ is some convex set defined at every point $p \in \Omega$.

${ }^{3}$ Sources and sinks can also be placed inside the domain. They would correspond to points in $\Omega$ where $\operatorname{div} \bar{f}$ is non-null, t.e. where stream $\bar{f}$ has an in-flow or out-flow.
} 
sites $\mathcal{P}$ to labels $\mathcal{L}$. Let $f_{p}$ be the label assigned to site $p$ and $f$ be the collection of such assignments for all sites in $\mathcal{P}$.

We can use the same general form of energy (2) that was earlier introduced in the context of binary labeling problems. The terms $D_{p}(l)$ are derived from the observed data and it expresses the label preferences for each site $p$. The smaller the value of $D_{p}(l)$, the more likely is the label $l$ for site $p$. Since adding a constant to $D_{p}(l)$ does not change the energy formulation, we assume, without loss of generality, that $D_{p}(l)$ 's are nonnegative. The pairwise potential $V_{p q}\left(l_{p}, l_{q}\right)$ expresses prior knowledge about the optimal labeling $f$. In general, prior knowledge can be arbitrarily complex, but in graph cuts based optimization, we are essentially limited to different types of spatial smoothness priors. Typically $V_{p q}\left(l_{p}, l_{q}\right)$ is a nondecreasing function of $\left\|l_{p}-l_{q}\right\|^{4}$. Different choices of $V_{p q}\left(l_{p}, l_{q}\right)$ imply different types of smoothness, see Sections 5.1 and 5.2 .

\subsection{Exact Multi-Label Optimization}

In this section, we describe the only known case of exact multi-label minimization of energy (2) via graph cuts. The corresponding graph construction is not covered by the general theoretical result in [25], which applies to binary labeling cases only. We have to make the assumption that labels are linearly ordered. This assumption limits the applicability of the method. For example, it cannot be directly used for motion estimation, since motion labels are 2 dimensional and cannot be linearly ordered ${ }^{5}$. Without loss of generality, assume that labels are integers in the range $\mathcal{L}=\{1, \ldots, k\}$. Let $V_{p q}=\lambda_{p q}\left|f_{p}-f_{q}\right|$. Then the energy is:

$$
E(f)=\sum_{p \in \mathcal{P}} D_{p}\left(f_{p}\right)+\sum_{(p, q) \in \mathcal{N}} \lambda_{p q}\left|f_{p}-f_{q}\right|,
$$

In vision, [20,9] were the first to minimize energy (3) with a minimum cut on a certain graph $\mathcal{G}$. In fact, this graph is topologically similar to a graph of Roy\&Cox [32] where separating hypersurface on 3D manifold gives a stereo disparity map, see Section 4.3.

The graph is constructed as follows. As usual, vertices $\mathcal{V}$ contain terminals $s$ and $t$. For each site $p$, create a set of nodes $p_{1}, \ldots, p_{k-1}$. Connect them with edges $\left\{t_{1}^{p}, \ldots, t_{k}^{p}\right\}$, where $t_{1}^{p}=\left(s, p_{1}\right), t_{j}^{p}=\left(p_{j-1}, p_{j}\right)$, and $t_{k}^{p}=\left(p_{k-1}, t\right)$. Each edge $t_{j}^{p}$ has weight $K_{p}+D_{p}(j)$, where $K_{p}=1+(k-1) \sum_{q \in \mathcal{N}_{p}} \lambda_{p q}$. Here $\mathcal{N}_{p}$ is the set of neighbors of $p$. For each pair of neighboring sites $p, q$ and for each $j \in\{1, \ldots, k-1\}$, create an edge $\left(p_{j}, q_{j}\right)$ with weight $\lambda_{p q}$. Figure 6 illustrates the part of $\mathcal{G}$ which corresponds to two neighbors $p$ and $q$. For each site $p$, a cut on $\mathcal{G}$ severs at least one edge $t_{i}^{p}$. The weights for $t_{i}^{p}$ are defined sufficiently large so that the minimum cut severs exactly one of them for each $p$. This establishes a natural correspondence between the minimum cut and an assignment of a label to $p$. If the minimum cut severs edge $t_{i}^{p}$, assign label $i$ to $p$. It is straightforward to show that the minimum cut corresponds to the optimum $f[9]$.

\footnotetext{
${ }^{4}$ Here we used the norm $\|\cdot\|$ notation because, in general, $l_{p}$ may be a vector

${ }^{5}$ Iterative application of the algorithm described here was used for motion in [33]
} 


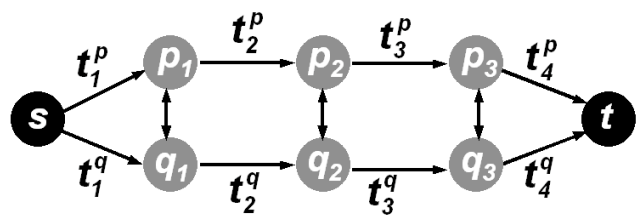

Fig. 6. Part of the graph construction for energy minimization in $3,|\mathcal{L}|=4$

Ishikawa [19] generalized the above construction to minimize any energy function with convex $V_{p q}$ 's. His construction is similar to the one in this section, except even more edges between $p_{i}$ 's and $q_{j}$ 's have to be added. Unfortunately, a convex $V_{p q}$ is not suitable for the majority of vision applications, especially if the number of labels is large. Typically, object properties tend to be smooth everywhere except the object boundaries, where discontinuities may be present. Thus in vision, a piecewise smooth model is more appropriate than the everywhere smooth model. However using a convex $V_{p q}$ essentially corresponds to the everywhere smooth model. The penalty that a convex $V_{p q}$ imposes on a sharp jumps in labels is so large, that in the optimal $f$ discontinuities are smoothed out with a "ramp". It is much cheaper to create a few small jumps in $f$ rather than one large jump. Of all the convex $V_{p q}$, the one in (3) works best for preserving discontinuities. Nevertheless in practice, it oversmooths disparity boundaries [38].

\subsection{Approximate Optimization}

The potential $V_{p q}$ in the previous section is not discontinuity preserving because $V_{p q}$ is allowed to grow arbitrarily large. One way to construct a discontinuity preserving $V_{p q}$ is to cap its maximum value. Perhaps the simplest example is the Potts model $V_{p q}=\lambda_{p q} \cdot \mathcal{I}\left(f_{p} \neq f_{q}\right)$ [10]. We have already seen Potts $V_{p q}$ in Section $3.1^{6}$, and it corresponds to the piecewise constant prior on $f$. Unfortunately, energy minimization with Potts $V_{p q}$ is NP-hard [10], however graph cuts can be used to find an answer within a factor of 2 from the optimum [10].

In this section, we describe two approximation methods, the expansion and the swap algorithms [10]. According to the results in [25], the swap algorithm may be used whenever $V_{p q}(\alpha, \alpha)+V_{p q}(\beta, \beta) \leq V_{p q}(\alpha, \beta)+V_{p q}(\beta, \alpha)$ for all $\alpha, \beta \in \mathcal{L}$, which we call the swap inequality. The expansion algorithm may be used whenever $V_{p q}(\alpha, \alpha)+V_{p q}(\beta, \gamma) \leq V_{p q}(\alpha, \gamma)+V_{p q}(\beta, \alpha)$ for all $\alpha, \beta, \gamma \in \mathcal{L}$, which we call the expansion inequality. Any $V_{p q}$ which satisfies the expansion inequality also satisfies the swap inequality, hence the expansion inequality is more restrictive.

Both swap and expansion inequalities admit discontinuity preserving $V_{p q}$ 's. The truncated linear $V_{p q}(\alpha, \beta)=\min (T,\|\alpha-\beta\|)$ satisfies the expansion inequality. The truncated quadratic $V_{p q}(\alpha, \beta)=\min \left(T,\|\alpha-\beta\|^{2}\right)$ satisfies the

\footnotetext{
${ }^{6}$ In the binary case, it is typically called the Ising model.
} 

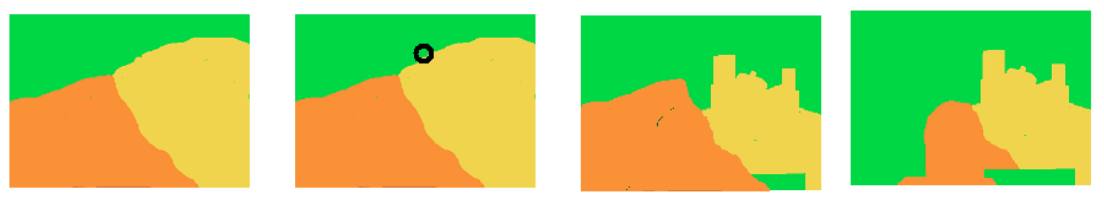

Fig. 7. From left to right: a labeling $f$, a labeling within one standard move of $f$ (the changed site is highlighted by a black circle), labeling within one green-yellow swap of $f$, labeling within one green expansion of $f$.

swap inequality. Here $T$ is a positive constant, which is the maximum penalty for a discontinuity. The truncated linear and truncated quadratic $V_{p q}$ correspond to a piecewise smooth model. Small deviations in labels incur only a small penalty, thus the smoothness is encouraged. However sharp jumps in labels are occasionally permitted because the penalty $T$ is not too severe to prohibit them.

Local Minimum with Respect to Expansion and Swap Moves Both the expansion and the swap algorithms find a local minimum of the energy function. However, in discrete optimization, the meaning of "a local minimum" has to be defined. For each $f$, we define a set of moves $M_{f}$. Intuitively, these are the moves to other labelings that are allowed from $f$. Then we say that $f$ is a local minimum with respect to the set of moves, if for any $f^{\prime} \in M_{f}, E\left(f^{\prime}\right) \geq E(f)$. Most discrete optimization methods (e.g. [13,3]) use standard moves, defined as follows. Let $H\left(f, f^{\prime}\right)$ be the number of sites for which $f$ and $f^{\prime}$ differ. Then for each $f$, standard moves are $M_{f}=\left\{f^{\prime} \mid H\left(f, f^{\prime}\right) \leq 1\right\}$. Thus a standard move allows to change a label of only one site in $f$, and hence $\left|M_{f}\right|$ is linear in the number of sites, making it is easy to find a local minimum with respect to the standard moves. The result, however is very dependent on the initial point since a high dimensional energy has a huge number of such local minima. In particular, the solution can be arbitrarily far from the global minimum.

We now define the swap moves. Given a labeling $f$ and a pair of labels $\alpha$ and $\beta$, a move $f^{\alpha \beta}$ is called an $\alpha-\beta$ swap if the only difference between $f$ and $f^{\alpha \beta}$ is that some sites that were labeled $\alpha$ in $f$ are now labeled $\beta$ in $f^{\alpha \beta}$, and some sites that were labeled $\beta$ in $f$ are now labeled $\alpha$ in $f^{\alpha \beta} . M_{f}$ is then defined as the collection of $\alpha-\beta$ swaps for all pairs of labels $\alpha, \beta \in \mathcal{L}$.

We now define the expansion moves. Given a labeling $f$ and a label $\alpha$, a move $f^{\alpha}$ is called an $\alpha$-expansion if the only difference between $f$ and $f^{\alpha}$ is that some sites that were not labeled $\alpha$ in $f$ are now labeled $\alpha$ in $f^{\alpha} . M_{f}$ is then defined as the collection of $\alpha$-expansions swaps for all labels $\alpha \in \mathcal{L}$. Figure 7 shows an example of standard move versus $\alpha$-expansion and $\alpha-\beta$ swap. Notice that a standard move is a special case of an $\alpha$-expansion and a $\alpha-\beta$ swap. However there are $\alpha$-expansion moves which are not $\alpha-\beta$ swaps and vice versa. 
The expansion (swap) move algorithm finds a local minimum with respect to expansion (swap) moves. The number of expansion (swap) moves from each labeling is exponential in the number of sites. Thus direct search for an optimal expansion (swap) move is not feasible. This is where graph cuts are essential. It is possible to compute the optimal $\alpha$-expansion or the optimal $\alpha-\beta$ swap with the minimum cut on a certain graph. This is because computing an optimal $\alpha$ expansion (optimal $\alpha-\beta$ swap) is a binary minimization problem which happens to be regular [25] when the expansion (swap) inequality holds.

The expansion (swap) algorithms are iterative. We start with an initial labeling $f$. We then cycle in random order until convergence over all labels $\alpha \in \mathcal{L}$ (pairs of $\alpha, \beta \in \mathcal{L}$ ), find the optimal $f^{\alpha}\left(f^{\alpha \beta}\right)$ out of all $\alpha$-expansions $(\alpha-\beta$ swaps), and change current labeling to $f^{\alpha}\left(f^{\alpha \beta}\right)$. Obviously this cannot lead to an increase in energy, and at convergence we found the local minimum with respect to expansion (swap) moves. Thus the key step is how to find the optimal $\alpha$-expansion ( $\alpha-\beta$ swap), which is performed by finding a minimum cut on a certain graph $\mathcal{G}=(\mathcal{V}, \mathcal{E})$. The actual graph constructions can be found in [10].

The criteria for a local minimum with respect to the expansions (swaps) are so strong that there are significantly fewer of such minima in high dimensional spaces compared to the standard moves. Thus the energy function at a local minimum is likely to be much lower. In fact, it can be shown that the local minimum with respect to expansion moves is within a constant factor of optimum. The best approximation is in case of the Potts model, where this factor is 2. It is not surprising then that most applications based on graph cuts use the expansion algorithm with the Potts model $[9,4,23,24,39,26,27,18,1,40]$.

\section{References}

1. M. Agrawal and L. Davis. Window-based, discontinuity preserving stereo. In CVPR04, pages I: 66-73, 2004.

2. B. Appleton and H. Talbot. Globally optimal surfaces by continuous maximal flows. In Digital Image Computing: Techniques and Applications, Proc. VIIth APRS, volume 1, pages 623-632, December 2003.

3. J. Besag. On the statistical analysis of dirty pictures (with discussion). Journal of the Royal Statistical Society, Series B, 48(3):259-302, 1986.

4. S. Birchfield and C. Tomasi. Multiway cut for stereo and motion with slanted surfaces. In ICCV99, pages 489-495, 1999.

5. A. Blake, C. Rother, M. Brown, P. Perez, and P. Torr. Interactive image segmentation using an adaptive gmmrf model. In 8th European Conference on Computer Vision, volume I of LNCS 3021, pages 428-441, Prague, Czech Republic, May 2004. Springer-Verlag.

6. Y. Boykov and G. Funka-Lea. Optimal Object Extraction via Constrained GraphCuts. International Journal of Computer Vision (IJCV), 2005, to appear. (Earlier version is in ICCV'01, vol. I, pp. 105-112, July 2001).

7. Y. Boykov and V. Kolmogorov. Computing geodesics and minimal surfaces via graph cuts. In International Conference on Computer Vision, volume I, pages 26-33, 2003. 
8. Y. Boykov and V. Kolmogorov. An experimental comparison of min-cut/maxflow algorithms for energy minimization in vision. IEEE Transactions on Pattern Analysis and Machine Intelligence, 26(9):1124-1137, September 2004.

9. Y. Boykov, O. Veksler, and R. Zabih. Markov random fields with efficient approximations. In IEEE Conference on Computer Vision and Pattern Recognition, pages 648-655, 1998.

10. Y. Boykov, O. Veksler, and R. Zabih. Fast Approximate Energy Minimization via Graph Cuts. IEEE Transactions on Pattern Analysis and Machine Intelligence, 23:1222-1239, 2001.

11. W. Cook, W. Cunningham, W. Pulleyblank, and A. Schrijver. Combinatorial Optimization. John Wiley \& Sons, 1998.

12. L. Ford and D. Fulkerson. Flows in Networks. Princeton University Press, 1962.

13. S. Geman and D. Geman. Stochastic relaxation, Gibbs distributions, and the Bayesian restoration of images. IEEE Transactions on Pattern Analysis and Machine Intelligence, 6:721-741, 1984.

14. A. Goldberg and R. Tarjan. A new approach to the maximum-flow problem. Journal of the Association for Computing Machinery, 35(4):921-940, October 1988.

15. L. Grady. Space-Variant Computer Vision: A Graph-Theoretic Approach. PhD thesis, Boston University, Boston, MA, 2004.

16. L. Grady and G. Funka-Lea. Multi-label image segmentation for medical applications based on graph-theoretic electrical potentials. In M. Šonka, I. A. Kakadiaris, and J. Kybic, editors, Computer Vision and Mathematical Methods in Medical and Biomedical Image Analysis, ECCV 2004 Workshops CVAMIA and MMBIA, number LNCS3117 in Lecture Notes in Computer Science, pages 230-245, Prague, Czech Republic, May 2004. Springer.

17. D. Greig, B. Porteous, and A. Seheult. Exact maximum a posteriori estimation for binary images. Journal of the Royal Statistical Society, Series B, 51(2):271-279, 1989.

18. L. Hong and G. Chen. Segment-based stereo matching using graph cuts. In CVPR04, pages I: 74-81, 2004.

19. H. Ishikawa. Exact optimization for Markov Random Fields with convex priors. IEEE Transactions on Pattern Analysis and Machine Intelligence, 25(10):13331336, 2003.

20. H. Ishikawa and D. Geiger. Occlusions, discontinuities, and epipolar lines in stereo. In 5th European Conference on Computer Vision, pages 232-248, 1998.

21. H. Ishikawa and D. Geiger. Segmentation by grouping junctions. In IEEE Conference on Computer Vision and Pattern Recognition, pages 125-131, 1998.

22. D. Kirsanov and S. Gortler. A discrete global minimization algorithm for continuous variational problems. Harvard Computer Science Technical Report, TR-14-04, July 2004, (also submitted to a journal).

23. V. Kolmogorov and R. Zabih. Computing visual correspondence with occlusions via graph cuts. In International Conference on Computer Vision, July 2001.

24. V. Kolmogorov and R. Zabih. Multi-camera scene reconstruction via graph cuts. In 7th European Conference on Computer Vision, volume III of LNCS 2352, pages 82-96, Copenhagen, Denmark, May 2002. Springer-Verlag.

25. V. Kolmogorov and R. Zabih. What energy functions can be minimized via graph cuts. IEEE Transactions on Pattern Analysis and Machine Intelligence, 26(2):147159, February 2004.

26. V. Kwatra, A. Schodl, I. Essa, and A. Bobick. GraphCut textures: image and video synthesis using graph cuts. In ACM Transactions on Graphics (SIGGRAPH), volume 22, July 2003. 
27. M. Lin and C. Tomasi. Surfaces with occlusions from layered stereo. PAMI, 26(8):1073-1078, August 2004.

28. S. Osher and R. Fedkiw. Level Set Methods and Dynamic Implicit Surfaces. Springer Verlag, 2002.

29. S. Osher and N. Paragios. Geometric Level Set Methods in Imaging, Vision, and Graphics. Springer Verlag, 2003.

30. C. Rother, V. Kolmogorov, and A. Blake. Grabcut - interactive foreground extraction using iterated graph cuts. In ACM Transactions on Graphics (SIGGRAPH), August 2004.

31. S. Roy. Stereo without epipolar lines: A maximum-flow formulation. International Journal of Computer Vision, 34(2/3):147-162, August 1999.

32. S. Roy and I. Cox. A maximum-flow formulation of the n-camera stereo correspondence problem. In IEEE Proc. of Int. Conference on Computer Vision, pages 492-499, 1998.

33. S. Roy and V. Govindu. MRF solutions for probabilistic optical flow formulations. In International Conference on Pattern Recognition (ICPR), September 2000.

34. G. Sapiro. Geometric Partial Differential Equations and Image Analysis. Cambridge University Press, 2001.

35. J. Sethian. Level Set Methods and Fast Marching Methods. Cambridge University Press, 1999.

36. G. Strang. Maximal flow through a domain. Mathematical Programming, 26:123$143,1983$.

37. M. Tappen and W. Freeman. Comparison of graph cuts with belief propagation for stereo, using identical mrf parameters. In IEEE Intl. Conference on Computer Vision (ICCV), October 2003.

38. O. Veksler. Efficient Graph-based Energy Minimization Methods in Computer Vision. PhD thesis, Cornell University, Ithaca, NY, August 1999.

39. J. Wills, S. Agarwal, and S. Belongie. What went where. In CVPR03, pages I: 37-44, 2003.

40. J. Xiao and M. Shah. Motion layer extraction in the presence of occlusion using graph cut. In CVPR04, pages II: 972-979, 2004.

41. N. Xu, R. Bansal, and N. Ahuja. Object segmentation using graph cuts based active contours. In IEEE Conference on Computer Vision and Pattern Recognition, volume II, pages 46-53, 2003. 